Contribuţii Botanice - 2017, LII: 23-28

DOI: $10.24193 /$ Contrib.Bot.52.2

Grădina Botanică "Alexandru Borza"

Cluj-Napoca

\title{
ANALYSIS OF FAMILY PRIMULACEAE FROM CARL STUDNICZKA'S HERBARIUM
}

\author{
Dalibor VLADOVIĆ ${ }^{1}$, Božena MITIĆ \\ ${ }^{1}$ Natural History Museum and Zoo, Kolombatovićevo šetalište 2, HR-21 000 Split, Croatia \\ ${ }^{2}$ Botanic Institute of PMF University of Zagreb, Marulićev trg 20/ II, HR-10 000 Zagreb, Croatia \\ e-mail: dalibor@prirodoslovni.hr
}

\begin{abstract}
In the C. Studniczka's herbarium we have found new herbarium folder which contain herbarium samples from family Primulaceae (Ord. Primulaceeen) which one part has already been analysed (Vladović et al., 2014b). We analysed the data from the new map and integrated with already known data for the family Primulaceae. Most of the analysed herbal material (95 herbarium sheets, with 391 samples of herbal plants) in this part of the C. Studniczka's herbarium were collected in Europe ( 87 herbarium sheets). According to the labels, the majority of herborized material was collected in the area of Austria (27 herbarium sheets). Most herbarium sheets belong to Flora Dalamtiens collection (11) - including Flora Süddalmatiens collection. In reference to the part of Studniczka's herbarium which has already been analysed, there is one botanist or collector which is mentioned for the first time: Hieronymus. Most herbarium sheets were collected by Studniczka himself (38). The oldest herbarium sheets dates from 1866, where as the newest ones date from 1904. The exact year of collection is missing from 6 herbarium labels.
\end{abstract}

Keywords: Studniczka's herbarium, Natural History Museum Split, Croatia.

\section{Introduction}

Since 2005, we have been working on Carl Studniczka's herbarium which can be found in the Natural History Museum in Split. As a botanist amateur, Carl Studniczka collected plants while he was traveling (1869-1904) and working as an officer (data are known from herbarium labels: wide surrounding area of Vienna and Wiener Neustadt in Austria; of Split, Hvar and Dubrovnik in Croatia; of Celje, Koper and Izola in Slovenia; of Kotor and Herceg Novi in Montenegro; of Olomouc and Leitmeritz in the Czech Republic; of Przemysl in Poland and at last the wide surrounding area of Trieste in Italy). The analysis has shown that this herbarium contains samples of the plants which were collected and sent to Studniczka by many botanists or collectors of that time.

\section{Material and Methods}

In this paper we analysed the herbarium sheet from family Primulaceae. From the labels of the herbarium sheets first, we copied the Latin name of the genus and the species; then the date, month and year of collection; followed by the collector (A-Ayasse, Al-Alioth, AuAusserdorfer, B-Baenitz, Br-Brandmayer, Be-Brendel, Bo-Bordère, E-Eggert, En-Engelmann, F-Freyn, G-Gandoger, Gr-Graf, H-Halácsy, Hi-Hieronymus, Hu-Huter, H/P-Huter et Porta, KKörnicke, Kr-Kristof， Ku-Kumlien， L-Lagger， Li-Lievre， R-Ressmann， Ri-Richter， SStudniczka, Sc-Schemmann, St-Strobl, T-Tauscher, W-Wiesbaur, Z-Zetterstedt, Z/W-Zetterstedt et Wickbom) and the affiliation to the particular herbarium collection (F-Flora Algeriensis 
exsiccata, FA-Flora Austriae inf., FAu-Flora Austriaca, FB- Flora Böhmens, FC-Flora Carinthiaca, FD-Flora Dalmatiens, FG-Flora Gallica exsiccata, FH-Flora Hungarica, FHe-Flora helvetica, FI-Flora Istrien, FN-Flora von Nieder- Oesterreich, FO-Flora von Obersteiermark, FPFlora von oesterr. Polen, FR-Flora von Russland, FS-Flora Süddalmatiens, FT-Flora von Triest, FW-Flora von Wien, FWr-Flora von Wr Neustadt, H-Eggert, Herbarium Americanum, HE-C. Baenitz, Herbarium Europaeum, HH-Herbarium Dr E. Halácsy, HS-Herbier de la Société d'Histoire Naturelle de Colmar, PV-Kumlien, Plante Visconsin, PS-Plante Scandinavicae, SSocietas Helvetica). According to The Plant list and USDA plants Database we listed the name of the species in brackets; from the label we copied the numeric sign, name of the place where the plant had been found with altitude data if available and listed the number of herbarium samples in brackets.

\section{Results and Discussion List of species}

1. Aletris farinosa L. En (A. farinosa L.) Louisiana (1) fam. Nartheciaceae

2. Androsace chamaejasme Host 7.1877. H HH (A. chamaejasme Wulfen) In pascuis alpinis montis Schneeber 4-5000' solo calc. (4)

3. A. elongata L. 8.4.1873. F (A. elongata L.) Siebenbürgen. Brachäcker bei Klausenburg Fert. Fegel 360 $\mathrm{m}(7)$

4. A. elongata L. 8.4.1882. S FB (A. elongata L.) sandige Hügel ober Schüttenitz (7)

5. A. glacialis Hoppe 12.8.1873. H/P (A. alpina/L./ Lam.) Lombardia. dit. Bresciana in mte Frerone vallis di Bagolino sol. granit. 7000' (9)

6. A. helvetica Gaud. 10.6.1866. Al (A. helvetica /L./ All.) Klimsenhorn, Pilatus (12)

7. A. lactea 19.6.1868. Hi FHe (A. lactea L.) Stock horn im Berner Oberland (7)

8. A. lactea L. 6.1873. Br FAu (A. lactea L.) am Almensattel aüf steig züm Schneeberg bei Puchberg (15)

9. A. lactea L. 13.7.1899. S FWr (A. lactea L.) am Schneeberg 6000' (2)

10. A. maxima L. 20.4.1893. S FN (A. maxima L.) Aecker üm Wr Neustadt (6)

11. A. obtusifolia All. 28.6.1869. Li (A. obtusifolia All.) Tirol, Schlern, alpentriften dolomit 6-7000' (4)

12. A. obtusifolia All. 7.1875. R FC (A. obtusifolia All.) Wolliggeralpe (2)

13. A. occidentalis Pursh. 25.4.1874. E H (A. occidentalis Pursh) Am Missisippi b. Cahokia. Ills (5)

14. A. septentrionalis L. 6. K FR (A. septentrionalis L.) Ufer der Ochta bei Petersburg (3)

15. A. septentrionalis L. 24.5.1872. S FW (A. septentrionalis L.) am sooser Lind kogel (4)

16. A. septentrionalis L. 14.5.1882. S FB (A. septentrionalis L.) sandige Waldplatze im Travčice Wald nächst Theresienstadt (10)

17. A. tauscheri Gandoger 20.5.1875. T SH (A. villosa L.subsp. taurica/Ovcz./ Fed.) Hungaria centralis Comitatus Alba E graminosis montanis prope Kulyavár (5)

18. A. tauscheri Gand 5.1877. Ri FH (Ex herb. Richter) (A. villosa L.subsp. taurica /Ovcz./ Fed.) Ort Ofen Blocksberg Unterlage dolomit Höhe 70 m (5)

19. A. villosa L. 15.7.1869. Kr (A. villosa L.) Hoch obir in Kärnten, über 6500-6700' (4)

20. A. villosa L. $\beta$ virescens 8.8.1899. S FWr (A. villosa L.) am Kaiserstein das Schneeberges 6800' (11)

21. Ammannia humilis Michx. 22.9.1875. E H (Rotala ramosior/L./ Koehne) Missisippithal in Ills. (4) fam. Lythraceae

22. A. latifolia L. 20.9.1875. E H (A. latifolia L.) An Blüffs Eisenbahn in Ills. (1) fam. Lythraceae

23. Aretia pennina Gaud. L (Androsace alpina/L./ Lam.) In monte Gries in Valeria superiori. (2)

24. Comandra umbellata Nutt.-Griseb. Ku PV (C. umbellata/L./ Nutt.) 152. Albion, Dane Countr. (2) fam. Santalaceae

25. Coris monspeliensis L. 1879. G F (C. monspeliensis L.) No 928 Hab. in ditione urbis Alger, loco dicto Mustapha (4)

26. Corthusa mathioli L. 6.1871. Au (Primula matthioli /L./ K.Richt.) Tirol austr. orient. ad terrentes et scaturigines in vallibur Virgen et Praegraten 5-5500' solo schistaceo calcar. Mixto (2)

27. Cortusa mathioli L. 6.1873. Br FN (Primula matthioli/L./ K.Richt.) Am Obersberge in der 
Schwarzau im Gebirge (2)

28. Cyclamen europaeum L. 22.7.1893. S FN (C. purpurascens Mill.) Wälder üm Wr Neustadt /:Kalkofen Malleiten:/ (3)

29. C. europaeum L. 12.8.1897. S FWr (C. purpurascens Mill.) hohe Wand (2)

30. C. europaeum L. 14.10.1902. S FT (C. purpurascens Mill.) in Gebüschen an der Napoleonstrasse ober Barcola (1)

31. $C$. hederaefolium Ait. $=C$. neapolitanum Ten. 9.1876. S FD $(C$. hederifolium Aiton) zwischen Gestrüpp, felsigen Orten um Castelnuovo (2)

32. C. neapolitanum Ten 11.1875. S FS (C. hederifolium Aiton) Villa Roitsch bei Spalato (2)

33. C. vernum Reich. $=$ C. repandum Sibth \& Sm 5.1878. S FD (C. repandum Sm.) Gesträüch ü. felsige Orte won dem dorfe Leševice in der Zuppa gegen Traste (3)

34. Glaux maritima L. 27.7.1871. B (Lysimachia maritima/L./ Galasso, Banfi \& Soldano) Dänzig: Strand bei Weichselmünde (6)

35. Hottonia palustris L. 5.1882. S FB (H. palustris L.) an der alten Eger bei Theresienstadt (5)

36. Hydrolea affinis Gray Be (H. uniflora Raf.) Palester Cv. Illinois (1) fam. Hydroleaceae

37. Ludwigia alternifolia L. 10.8.1875. E H (L. alternifolia L.) Gräben hinter d. Forest Park (1) fam. Onagraceae

38. Primula acaulis Jacq. 5.2.1873. S FD (P. vulgaris Huds.) in feuchten Gebüschen um Castelnuovo (3)

39. $P$. acaulis Jacq. $=P$. grandiflora Lam. 4.1879. A (P. vulgaris Huds.) Les haies et bois à Genéve (2)

40. P. acaulis Jacq. 20.4.1901. S FT (P. vulgaris Huds.) im Wald won Rojano (7)

41. P. auricula L. 18.4.1871. Br FAu (P. auricula L.) felsen des Maaberges bei Mödling (2)

42. P. clusiana Tausch 1.6.1873. Br FAu (P. clusiana Tausch) Am Obersberge in der Schwarzau im Gebirge (5)

43. P. clusiana Tausch 6.1878. H FA (P. clusiana Tausch) In petrosis alpinis mt. Raxalpe 5-6000' (4)

44. P. comutata Schott. 4.1876. Gr (P. villosa Wulfen) Stiria. St. Johann bei Herberstein (3)

45. P. elatior Jcq. 4.1873. H FA (P. elatior/L./ Hill) In pratis mont. ad Rekawinkel (3)

46. P. elatior L. 4.1885. S FP (P. elatior/L./ Hill) mehr sonnige Hügel um Przemysl (3)

47. P. elatior Jacq. f. brachyphylos 9.4.1873. Sc HE (P. elatior/L./ Hill) No 3662 Annen. Wiesen (3)

48. P. elatior Jacq. f. macrostylos 17.4.1874. Sc HE (P. elatior/L./ Hill) No 3663 Annen. Wiesen (3)

49. P. farinosa L. 14.5.1872. S FW (P. farinosa L.) sumpfge Wiesen zu Laxenburg, Achau, Himberg \& Velm. (2)

50. P. farinosa L. 15.5.1894. S FN (P. farinosa L.) Sümpfwiesen bei Stollhof (neür Wald) (6)

51. P. glutinosa L. 8.1873. Gr (P. glutinosa Wulfen) Stiria. Hochzinken 7500' (1)

52. P. integrifolia L. 5.1873. Br FN (P. integrifolia L.) Schwaben wiese Puchberg (4)

53. P. intricata G.G. 6. Bo HS (P. elatior/L./ Hill subsp. intricata/Gren. \& Godr./ Widmer) Localité Gavarnie Htes Pyr, Station paturages froidi Altitude $1400 \mathrm{~m}$ Terrain calcair (5)

54. P. minima L. 9.7.1870. B (P. minima L.) Reisengebirge: kleiner Teich (5)

55. P. montana Opitz 3.4.1877. G FG (P. elatior/L./ Hill) No 990. Hab. in collibus prope Denicé (Rhône) (2)

56. P. officinalis Jacq. v. macrostylos Peterm. 18.5.1874. B HE (P. veris L.) Königsberg i Pr.: Wälder bei Schlobitten (2)

57. P. officinalis Jacq. 5.1882. S FB (P. veris L.) Kreutzberg bei Leitmeritz in Wäldern (2)

58. P. officinalis Scop. 9.5.1897. S FWr (P. veris L.) aüf d. Malleiten bei fischaü (3)

59. P. scotica Hook. 3.7.1870. Z/W (P. scotica Hook.) Norvegia: Dovre, Kongsvold in pratis uliginosis (3)

60. P. stricta Horn. 4.7.1868. Z (P. stricta Hornem.) Norge, Finmarken, Alten, Bosekop. (1)

61. P. stricta Horn. 27.6.1870. Z PS (P. stricta Hornem.) Dovre: Harbakken (2)

62. P. suaveolens Bertol. 13.5.1874. A (P. veris L. subsp. suaveolens /Bertol./ Gutermann \& Ehrend.) Garnié les buissons à Genthod prés Genéve (1)

63. P. suaveolens Bertol. 23.6.1875. S FD (P. veris L. subsp. suaveolens /Bertol./ Gutermann \& Ehrend.) im Wäldern des Biokovo 2-4000' (2)

64. P. suaveolens Bertol. 22.6.1879. S FD (P. veris L. subsp. suaveolens /Bertol./ Gutermann \& Ehrend.) Waldblössen am Vuči zub des Orien in der Krivošie 4-5000' (1)

65. P. tirolensis Schott $=P$. allionii Koch et Kern 18.6.1872. H (P. tyrolensis Schott) Venetia, dit. d' Agordo ad mtem Civetta pr. Alleghe loc. dict. Entrasass rup. dolomitic. 3400' (5) 
66. P. variabilis Goupil (P. offi. x acaulis) 18.4.1874. W HE (P. $\times$ polyantha Mill.) Kalksburg bei Wien. Park (6)

67. P. vulgaris Huds. $\beta$ caulescens Neilr. 4.1877. H FA (P. vulgaris Huds.) In silvis montis Auninger ad Viennam (2)

68. Samolus floridibus HB.K. 9.9.1875. E H (S. floribundus Kunth) Blüffs in Ills. (3)

69. S. valerandi L. 27.6.1874. S FS (S. valerandi L.) Sümpfe bei Stobrez (1)

70. S. valerandi L. 5.1878. S FD (S. valerandi L.) quellige Orte nächst Glavati bei Cattaro (3)

71. S. valerandi L. 15.6.1904. S FI (S. valerandi L.) in Gräben längst der Bahn von Semedella (5)

72. Soldanella alpina L. 6. Br FN (S. alpina L.) Schneeberg (5)

73. S. minima Hpp. 29.4.1869. St FO (S. minima Hoppe) In der Waldregion um Admont Kälkshütt aüf der Büchaü 3000' (5)

74. Triosteum perfoliatum L. 16.6.1875. E H (T. perfoliatum L.) hinter d. Schülzenpark (2) fam. Caprifoliaceae

Total number of the herbarium scheets in analysed family is 95 (with 391 samples of herbal plants). The majority of the plants was collected in Europe ( 87 herbarium sheets), 6 sheets originate from North America and two sheets originate from Africa.

Most of the herbarium samples were collected in the area of Austria (27 herbarium sheets) and: Italy (9), Poland (8), Czech Republic (7), Germany (7), Montenegro (6), USA (6), Croatia (5), France (4), Switzerland (4), Norway (3), Algeria (2) Romania (2) and Slovenia (2). One herbarium sheet was collected in: Belgium, Hungary and Russia. According to the affiliation to a particular herbarium collection in analysed familys or their parts the most represented is Flora Dalmatiens (including Flora Süddalmatiens collection) with 11 herbarium sheets. This is followed by other collections, such as: Flora Böhmens (6), C. Baenitz Herbarium Europaeum (6), Flora von N. Oesterreich (6) - including Flora von Nieder- Oesterreich collection, Flora von Triest (5), Flora von Wr. Neustadt (5), Flora von oesterr. Polen (4), Eggert Herbarium Americanum (4) and others. There are 19 herbarium sheets that are unmarked and therefore we do not know which herbarium collection they belong to.

Most of the samples of herbarium plants were collected by Studniczka himself (38 herbarium sheets), while others were sent to him by: Brandmayer (6), Gandoger (4), Halácsy (4), Eggert (4), Baenitz (3), Körnicke (3), Schemmann (3) and others. In the part of the herbarium which has already been analysed (compare with 1, 2, 3, 4, 5, 6, 9, 10, 11, 12, 13, 14, 15, 16, 17, $18,19,20,21,22,23,24,25,26,27,28,29,30,31,32,33,34,35,36)$, there is one botanist or collector of the herbal material which is mentioned for the first time: Hieronymus.

The oldest herbarium sheet dates from 1866, where as the newest ones date from 1904. The vast majority of herbarium sheets (56), was collected during the period from 1870 to 1880 . The exact year of collection is missing from 6 herbarium labels.

According to Studniczka, within 95 herbarium sheets (order Primulacen) there are 15 genera with 59 species, and 7 varietas.

\section{REFERENCES}

1. Mitić, B., Vladović, D., Ževrnja, N., Anterić, P., 2008, Analysis of Ord. Berberideen, Nymphaeaceen, Papaveraceen and Fumariaceen from C. Studniczka`s Herbarium. Hladnikia, Ljubljana, 22: 61.

2. Mitić, B., Vladović, D., Ževrnja, N., Anterić, P., 2008, Analysis of Ord. Berberideen, Nymphaeaceen, Papaveraceen \& Fumariaceen from C. Studniczka`s Herbarium, Prirodoslovni muzej i zoološki vrt, Split. 
3. Mitić, B., Vladović, D., Ževrnja, N., 2010-2011, Crassulaceen, Marsileaceen i Saxifragaceen iz herbara C. Studniczke. The Crassulaceen, Marsileaceen and Saxifragaceen - Collection of the herbarium C. Studniczke, Radovi hrvatskoga društva za znanost i umjetnost, Sarajevo, XII-XIII: 42-48..

4. Mitić, B., Vladović, D., Ževrnja, N., 2011, Analysis of Family Rosaceae from Carl Studniczka`s Herbarium, Natura Montenegrina, 10 (2): 71-85.

5. Mitić, B., Vladović, D., Ževrnja, N., 2012, Analysis of some Familys from Carl Studniczka's Herbarium (I). Natura Montenegrina, 11 (3): 397-404.

6. Mitić, B., Vladović, D., Ževrnja, N., 2013, Ord. Crassulaceen, Marsileaceen i Saxifragaceen iz herbarija C. Studniczke, Prirodoslovni muzej Split (Hrvatska), Prirodoslovni muzej i zoološki vrt, Split.

7. THE PLANT LIST www.theplantlist.org

8. USDA PLANTS DATABASE plants.usda.gov

9. Vladović, D., Ževrnja, N., Mitić, B., Tomasović, D., 2007a, The analysis of the order Ranunculales in the herbarium of C. Studniczka. In: Britvec M., Škvorc Ž. (ed.). Book of Abstracts, 2nd Croatian Botanical Congress, 20.-22. rujna 2007., Zagreb: 58-59.

10. Vladović, D., Ževrnja, N., Mitić, B., Tomasović, D., Bradarić, D., 2007b, Die Analyse ord. Ranunculaceen aus dem Herbarium von C. Studniczka, Prirodoslovni muzej i zoološki vrt, Split.

11. Vladović, D., Ževrnja, N., Mitić, B., 2009a, Analysis of Ord. Violarieen, Capparideen, Cistineen and Sileneen from C. Studniczka's herbarium.. In: Bacchetta G. (ed.). Book of Abstracts, Biodiversity Hotspots in the Mediterranean Area, 45th International Congress of SISV \& FIP Cagliari 22-24/25-29 June 2009, p. 104.

12. Vladović, D., Ževrnja, N., Mitić, B., 2009b, L'analisi delle Ord. Violarieen, Capparideen,Cistineen e Sileneen dall' erbario di C. Studnitzka, Prirodoslovni muzej i zoološki vrt, Split.

13. Vladović, D., Ževrnja, N., Mitić, B., 2010a, The Papilionaceen-collection of the Herbarium C. Studniczka. Natural History Museum Split (Croatia). In: Dolenc Koce, J. et al. (ed.). Book of Abstracts, 5th Slovenian symposium on plant biology, September 6-9, 2010, Ljubljana, p. 50.

14. Vladović, D., Ževrnja, N., Mitić, B., 2010b, The Papilionaceen-collection of the Herbarium C. Studniczka, Natural History Museum Split (Croatia), Prirodoslovni muzej i zoološki vrt, Split.

15. Vladović, D., Ževrnja, N., Mitić, B., 2011, Analysis of family Umbelliferae form C. Studniczka's herbarium. In: Biondi E. et al. (ed.). Book of programme, abstracts, excursion guide, 34th International Symposium of the Eastern Alpine and Dinaric Society for Vegetation Ecology, May 24-28, 2011., Camerino (Marches, central Italy), p.51.

16. Vladović, D., Ževrnja, N., Mitić, B., 2012, Analiza redova „Alsineen“, „Xanthoxyleen“ i „Elatineen“ iz herbarija C. Studniczke. In: Jelaska S.D. et al (ed.). Book of Abstracts, 11th Croatian Biological Congess, 16th.-21th. september, Šibenik, p.37.

17. Vladović, D., Mitić, B., Ževrnja, N., 2013a, Analysis of some Families from Carl Studniczka's Herbarium (III). In: AlegroA. \& Boršić I. (ed.). Book of Abstracts 4th Croatian Botanical Symposium with international participation 27 - 29 September 2013 Split, Croatia: 128-129.

18. Vladović, D., Ževrnja, N., Mitić, B., 2013b, The Umbelliferae - Collection of the herbarium Carl Studniczka, Prirodoslovni muzej i zoološki vrt, Split.

19. Vladović, D., Mitić, B., Ževrnja, N., 2014a, Analysis of the Family Labiaceae from Carl Studniczka's herbarium, Bul. Grăd. Bot. Cluj, XLIX: 85-96.

20. Vladović, D., Mitić, B., Ževrnja, N., 2014b, Analiza Ord. Acanthaceen, Ericineen, Primulaceen i Vaccinieen iz herbarija C. Studniczke, Naš krš Sarajevo, XXXIV, 47: 200-221.

21. Vladović, D., Mitić, B., Ževrnja, N., 2015, Addition to the family Apiaceae from Carl Studniczka's herbarium, Book of Abstracts 14.-18. September 2015. 6th Balkan Botanical Congress International Symposium, Rijeka, p 121.

22. Vladović, D., Mitić, B., 2016, Analysis of the Family Boraginaceae from Carl Studniczka's herbarium, Acta Horti Bot. Bucurest., 43: 57-66.

23. Ževrnja, N., Mitić, B., Vladović, D., Anterić, P., 2008a, Analyse Ord. Cruciferen aus dem C. Studniczka Herbar, Sauteria, 16: 415-416.

24. Ževrnja, N., Mitić, B., Vladović, D., Anterić, P., 2008b, Analysis of Ord. Cruciferen from C. Studniczka`s Herbarium, Prirodoslovni muzej i zoološki vrt, Split. 
25. Ževrnja, N., Mitić, B., Vladović, D., Anterić, P., 2009a, Die Cruciferen-Sammlung des Herbariums von C. Studniczka im Naturkundemuseum Split (Kroatien), Sauteria, 18: 299-307.

26. Ževrnja, N., Mitić, B., Vladović, D., 2009, Analysis of Ord. Geraniaceen, Oxalideen, Rutaceen and Lineen from C. Studniczka`s herbarium. In: Ninov, N. (ed.). Book of Abstracts, International scientific conference Balkans, Hot Spots of Ancient and Present Genetic Diversity, 17-20 june 2009., Sofia, Bulgaria, p.60.

27. Ževrnja, N., Mitić, B., Vladović, D., 2009b, Analysis of Ord. Geraniaceen, Oxalideen, Rutaceen and Lineen from C. Studniczka`s herbarium, Prirodoslovni muzej i zoološki vrt, Split.

28. Ževrnja, N., Mitić, B., Vladović, D., 2010a, The new findings from C. Studniczkas herbarium. In: Jasprica N. et al (ed.). Book of Abstracts, 3rd Croatian Botanical Congess, 24.-26. rujna, otok Murter, p. 210-211.

29. Ževrnja, N., Mitić, B., Vladović, D., 2010b, Analysis of Ord. Hippocastanēn, Balsimeinēn, Acerineen, Ampelideen, Malvaceen and Hypericineen from C. Studniczka's herbarium, Prirodoslovni muzej i zoološki $v r t$, Split.

30. Ževrnja, N., Mitić, B., Vladović, D., 2011, Novosti iz herbarija Carla Studniczke (Sanguisorbeen, Pomaceen, Granateen), Prirodoslovlje 11 (1): 19-28.

31. Ževrnja, N., Mitić, B., Vladović, D., 2013a, Analysis of some Families From Carl Studniczka's Herbarium (II), Natura Montenegrina, 12 (1): 241-250.

32. Ževrnja, N., Mitić, B., Vladović, D., 2013b, News from C. Studniczka's herbarium (ord. Onagrarieen, Cucurbitaceen and Portulaceen). In: Ribeiro D. et al (ed.), Book of Abstracts, 35th Meeting of Eastern Alpine and Dinaric Society for Vegetation Ecology. Ohrid (Republic of Macedonia), 3.-6. 7. 2013, p. 4647.

33. Ževrnja, N., Mitić, B.,Vladović, D., Cvitanić, R., Mekinić, S., Boban, J., 2013, Analysis of some Familys From Carl Studniczka's Herbarium (IV). In: Pešić V. (ed.), Book of Abstracts, V International Symposium of Ecologist of the Republic of Montenegro, 2- 5- 10. 2013, Tivat, p.88.

34. Ževrnja, N., Mitić, B., Vladović, D., 2014a, Analysis of some familys from Carl Studniczka's herbarium (V). GZM (PN), NS, 35: 31-37.

35. Ževrnja, N., Mitić, B., Vladović, D., 2014b, Analiza Ord. Gentianeen, Convolvulaceen, i Ebenaceen iz herbarija C. Studniczke, Naš krš Sarajevo, XXXV, 48: 144-156.

36. Ževrnja, N., Mitić, B., Vladović, D., 2015, The analysis of part of Carl Studniczka's herbarium (ord. Lonicereen, Caprifoliaceen and Stellateen), Book of Abstracts, 12th Croatian Biological Congress with International Participation, 18. - 23. IX 2015, Sveti Martin na Muri, p. 178.

\section{ANALIZA FAMILIEI PRIMULACEAE DIN HERBARUL CARL STUDNICZA}

\section{(Rezumat)}

În herbarul C. Studniczka au fost găsite coli noi de herbar cu exemplare aparţinând familiei Primulaceae dintre care, o parte au fost deja analizate (Vladović et al., 2014b). Au fost studiate noile date şi apoi corelate cu informaţiile deja cunoscute pentru familia Primulaceae. Cele mai multe dintre colile de herbar analizate ( 95 coli, 391 de exemplare) din herbarul C. Studniczka, au fost colectate în Europa ( 87 coli de herbar). Conform etichetelor a rezultat că majoritatea exemplarelor au fost colectate din Austria (27 coli de herbar). Dintre acestea 11 coli de herbar aparţin Florei Dalmaţiei - incluzând aici colecţia din Flora Dalmaţiei de Sud. Referindu-ne la partea din herbarul C. Studniczka care a fost deja analizată observăm numele unui botanist sau culegator care este menţionat pentru prima dată: Hieronymus. Cele mai multe coli de herbar au fost colectate de C. Studniczka însuşi (38). Cele mai vechi coli de herbar datează din anul 1866, iar cele mai noi din anul 1904. La un număr de 6 coli de herbar nu se cunoaşte anul colectării. 